\title{
Genetic and phenotypic diversity in Burkholderia: contributions by prophage and phage-like elements
}

\author{
Catherine M Ronning ${ }^{1,4 \dagger}$, Liliana Losada ${ }^{1 \dagger}$, Lauren Brinkac ${ }^{1}$, Jason Inman ${ }^{1}$, Ricky L Ulrich², Mark Schell3 \\ William C Nierman", David DeShazer ${ }^{2^{*}}$
}

\begin{abstract}
Background: Burkholderia species exhibit enormous phenotypic diversity, ranging from the nonpathogenic, soiland water-inhabiting Burkholderia thailandensis to the virulent, host-adapted mammalian pathogen B. mallei. Genomic diversity is evident within Burkholderia species as well. Individual isolates of Burkholderia pseudomallei and B. thailandensis, for example, carry a variety of strain-specific genomic islands (Gls), including putative pathogenicity and metabolic islands, prophage-like islands, and prophages. These Gls may provide some strains with a competitive advantage in the environment and/or in the host relative to other strains.
\end{abstract}

Results: Here we present the results of analysis of 37 prophages, putative prophages, and prophage-like elements from six different Burkholderia species. Five of these were spontaneously induced to form bacteriophage particles from B. pseudomallei and B. thailandensis strains and were isolated and fully sequenced; 24 were computationally predicted in sequenced Burkholderia genomes; and eight are previously characterized prophages or prophage-like elements. The results reveal numerous differences in both genome structure and gene content among elements derived from different species as well as from strains within species, due in part to the incorporation of additional DNA, or 'morons' into the prophage genomes. Implications for pathogenicity are also discussed. Lastly, RNAseq analysis of gene expression showed that many of the genes in $\varphi 1026 \mathrm{~b}$ that appear to contribute to phage and lysogen fitness were expressed independently of the phage structural and replication genes.

Conclusions: This study provides the first estimate of the relative contribution of prophages to the vast phenotypic diversity found among the Burkholderiae.

\section{Background}

Burkholderia pseudomallei, causal agent of the potentially fatal disease melioidosis, is a metabolically versatile soil organism that has been classified as a Category B biological threat by the CDC [1,2]. Relatively little is known about its pathogenesis, virulence factors, the extent of diversity in natural populations, and host response. B. pseudomallei genome plasticity has been associated with genomic island variation. The genome of B. pseudomallei $\mathrm{K} 96243$ (7.3 Mb), for example, features 16 genomic islands, at least three of which appear to be prophages [3]. It is unclear whether all of these putative

\footnotetext{
* Correspondence: david.deshazer@amedd.army.mil

† Contributed equally

${ }^{2}$ U.S. Army Medical Research Institute of Infectious Diseases, 1425 Porter
} Street, Fort Detrick, MD 21702, USA prophages are active, although one (ФK96243) was shown to be a productive bacteriophage. B. pseudomallei isolates are genetically quite diverse $[4,5]$, and this heterogeneity may be due at least in part to the highly variable distribution of bacteriophages among strains [6]. Such differences may provide certain strains survival advantages in the environment and the host, as well as explain the variable clinical presentation of melioidosis.

Also raising concern as a potential biological weapon is the very closely related $B$. mallei, causal agent of the primarily equine disease known as glanders [7]. In contrast to $B$. pseudomallei, $B$. mallei is a highly specialized pathogen, not found outside of a mammalian host in nature. B. mallei is a host-adapted clone of B. pseudomallei, and all of the $B$. mallei genome is nearly identical to a set of genes within B. pseudomallei core genome. However, 
in addition to its core genome $B$. pseudomallei contains numerous contiguous gene clusters that were deleted from B. mallei during its evolution $[8,9]$.

B. thailandensis is another closely related organism often found in the same environmental samples (soil and water of endemic melioidosis regions) as B. pseudomallei [10]. Unlike B. pseudomallei and B. mallei, B. thailandensis has very low virulence in most animal hosts, including humans. The ability to metabolize arabinose, and the corresponding loss of the arabinose assimilation operon from $B$. pseudomallei, phenotypically distinguishes $B$. thailandensis from $B$. pseudomallei [11]. The genes encoding arabinose assimilation may be considered as antivirulent, and their absence from $B$. pseudomallei (and B. mallei) may have allowed the development of the latter as pathogens [12]. Burkholderia multivorans, a member of the Burkholderia cepacia complex, is an opportunistic pathogen associated with infection in cystic fibrosis patients that is also found in soil environments [13].

The presence of prophages among bacterial isolates and their possible contribution to bacterial diversity is widespread. By carrying various elements contributing to virulence, prophages can contribute to the genetic individuality of a bacterial strain. This phenomenon has been reported in Salmonella spp [14] and Lactobacillus spp $[15,16]$, among others. Prophage-associated chromosomal rearrangements and deletions have been found to be largely responsible for strain-specific differences in Streptococcus pyogenes [17] and Xylella fastidiosa [18]. Thus, temperate phages carrying foreign DNA can play a role in the emergence of pathogenic variants. Lateral gene transfer between phage and host genomes, and phage lysogenic conversion genes, can alter host phenotype through production of phage-encoded toxins and disease-modifying factors that affect virulence of the bacterial strain. Examples of such phage-encoded virulence factors abound in the literature, and include proteins associated with toxicity, antigenicity, invasion, intracellular survival, serum resistance, and adhesion [19]. Many of these factors are encoded by morons that are present variably across phage genomes and are thought to be regulated independently of the phage genes [20].

To estimate the contribution of prophages to genetic and phenotypic diversity of the species, we have isolated and sequenced five temperate bacgteriophages from Burkholderia, three from B. pseudomallei and two from B. thailandensis, and used bioinformatics techniques to search for putative prophage regions in the genomes of nine sequenced $B$. pseudomallei strains, six $B$. malle $i$ strains, one $B$. thailandensis strain, three B. multivorans strains, and one Burkholderia xenovorans strain. While no prophages were detected in any of the $B$. malle $i$ strains, a total of 24 putative prophages or prophage-like islands (PI) were identified in the other species. Sequences from the isolated phages and inferred prophages were compared with each other and with the 8 published phage sequences from B. pseudomallei, $B$. thailandensis, B. cenocepacia, and B. cepacia. As seen in other genera, the prophages among the Burkholderiae contribute to the genomic variability of the species and carry genes that could provide advantages in the environment and host adaptation.

\section{Methods}

Spontaneous bacteriophage production by lysogenic B. pseudomallei and $B$. thailandensis strains, host range studies, and UV induction experiments

Five bacteriophages were isolated and fully sequenced (Table 1A).

Bacteriophage production and plaque formation by $B$. pseudomalle $i$ and $B$. thailandensis strains were assessed using B. mallei ATCC 23344 as an indicator strain, as described previously [6,21].

B. pseudomallei strains Pasteur 52237, E12, and 644 and $B$. thailandensis strains E202 and E255 were grown in LB broth for $18 \mathrm{~h}$ at $37^{\circ} \mathrm{C}$ with shaking $(250 \mathrm{rpm}$ ). Overnight cultures were briefly centrifuged to pellet the cells, and the supernatants were filter-sterilized ( 0.45 $\mathrm{mm}$ ). The samples were serially diluted in suspension medium (SM) [22], and the number of plaque forming units (pfu) was assessed using B. mallei ATCC 23344 as the host strain. Briefly, one hundred microliters of filtersterilized culture supernatant was added to a saturated B. mallei ATCC 23344 culture, incubated at $25^{\circ} \mathrm{C}$ for 20 min, and $4.8 \mathrm{ml}$ of molten LB top agar (0.7\%) containing $4 \%$ glycerol was added. The mixture was immediately poured onto a LB plate containing $4 \%$ glycerol and incubated overnight at $25^{\circ} \mathrm{C}$ or $37^{\circ} \mathrm{C}$. For $\varphi \mathrm{E} 202$ host range studies, this procedure was followed using the bacteria listed in Additional file 1, Table S1. Bacteria were considered to be sensitive to $\varphi \mathrm{E} 202$ if they formed plaques under these conditions and resistant if they did not. No bacterial species tested formed plaques in the absence of $\varphi$ E202.

For $\varphi$ E202 UV induction studies, one hundred microliters of saturated $B$. thailandensis E202 culture was used to inoculate two LB broth $(3 \mathrm{ml})$ subcultures. One set of subcultures was incubated for $5 \mathrm{~h}$ without interruption. The other set of subcultures was incubated for $3 \mathrm{~h}$, poured into sterile petri dishes in a class II biological safety cabinet, subjected to a hand-held UV light source $(254 \mathrm{~nm})$ for $20 \mathrm{sec}(25 \mathrm{~cm}$ above the sample), pipetted back into culture tubes, and incubated for an additional $2 \mathrm{~h}$. The titer of the filter-sterilized supernatants were determined by performing quantitative plaque assays on serially diluted samples. 
Table 1 Sources and descriptions of bacteriophage and putative prophage islands (PI) used in this study.

\begin{tabular}{|c|c|c|c|c|c|c|c|c|}
\hline \multicolumn{9}{|l|}{$\begin{array}{l}\text { A. Isolated } \\
\text { bacteriophages }\end{array}$} \\
\hline Phage (Acc \#) & Source & Description & $\begin{array}{l}\text { Size } \\
(\mathrm{Mb})\end{array}$ & $\begin{array}{c}\# \\
\text { ORFs }\end{array}$ & $\begin{array}{c}\text { Head } \\
\text { diameter } \\
(\mathbf{n m})\end{array}$ & $\begin{array}{l}\text { Tail (length } \times \\
\text { diameter) }(\mathrm{nm})\end{array}$ & $\begin{array}{l}\text { Plaque } \\
\text { diameter } \\
(\mathrm{mm})\end{array}$ & $\mathrm{pfu} / \mathrm{mL}$ \\
\hline $\begin{array}{l}\varphi 52237 \\
(\text { NC_007145) }\end{array}$ & $\begin{array}{l}\text { Bp Pasteur } \\
52237\end{array}$ & & 37.6 & 47 & 55 & $155 \times 23$ & $1.5-2.0$ & $3 \times 10^{6}$ \\
\hline $\begin{array}{l}\varphi 644-2 \\
\text { (NC_009235) }\end{array}$ & Bp 644 & Australia; disease (ulcer) & 48.7 & 71 & 60 & $190 \times 9$ & 1.0 & $3 \times 10^{3}$ \\
\hline $\begin{array}{l}\varphi \text { E12-2 } \\
\text { (NC_009236) }\end{array}$ & Bp E12-2 & NE Thailand; soil & 36.7 & 50 & 62 & $152 \times 21$ & $1.5-2.0$ & $1 \times 10^{1}$ \\
\hline $\begin{array}{l}\varphi \text { E202 } \\
\text { (NC_009234) }\end{array}$ & Bt E202 & NE Thailand; soil & 35.7 & 48 & 65 & $140 \times 21$ & $1.5-2.0$ & $2 \times 10^{5}$ \\
\hline $\begin{array}{l}\varphi \mathrm{E} 255 \\
\left(\mathrm{NC} \_009237\right)\end{array}$ & Bt E255 & central Thailand; soil & 37.4 & 55 & 64 & $143 \times 21$ & 0.5 & $2 \times 10^{3}$ \\
\hline \multicolumn{9}{|l|}{$\begin{array}{l}\text { B. Inferred } \\
\text { prophages }\end{array}$} \\
\hline $\begin{array}{l}\text { Prophage-like } \\
\text { island }\end{array}$ & Source & ORFs & $\begin{array}{l}\text { Size } \\
(\mathrm{Mb})\end{array}$ & $\begin{array}{c}\# \\
\text { ORFs } \\
\end{array}$ & Chromosome & Description & & \\
\hline PI S13-1 & Bp S13 & $\begin{array}{l}\text { BURPSS13_G0002-G0044; } \\
\text { BURPSS13_I0965-10971 }\end{array}$ & 38.0 & 48 & । & putative prophage & & \\
\hline PI S13-2 & Bp S13 & $\begin{array}{l}\text { BURPSS13_T0353-T0354; } \\
\text { BURPSS13_K0001-K0007 }\end{array}$ & 9.5 & 9 & $\|$ & prophage-like & & \\
\hline PI S13-3 & Bp S13 & BURPSS13_T0561-T0598 & 23.4 & 38 & $\|$ & prophage-like & & \\
\hline PI Pasteur-2 & $\begin{array}{l}\text { Bp Pasteur } \\
6068\end{array}$ & BURPSPAST_Y0106-Y0135 & 42.4 & 30 & 1 & putative prophage & & \\
\hline PI Pasteur-3 & $\begin{array}{l}\text { Bp Pasteur } \\
6068\end{array}$ & BURPSPAST_P0245-P0287 & 60.1 & 45 & 1 & prophage-like & & \\
\hline PI 1655-1 & Bp 1655 & BURPS1655_F0102-F0150 & 36.9 & 48 & 1 & putative prophage & & \\
\hline PI 406E-1 & Bp 406E & BURPS406E_K0245-K0264 & 17.9 & 20 & । & putative prophage & & \\
\hline PI 406E-2 & Bp 406E & BURPS406E_R0182-R0256 & 62.9 & 73 & । & putative prophage & & \\
\hline PI 1710b-1 & Bp 1710b & BURPS1710B_1505-1536 & 47.0 & 32 & 1 & putative prophage & & \\
\hline PI 1710b-2 & Bp 1710b & BURPS1710B_1538-1604 & 61.1 & 67 & 1 & putative prophage & & \\
\hline PI 1710b-3 & Bp 1710b & BURPS1710B_3650-3669 & 63.0 & 45 & । & prophage-like & & \\
\hline PI 688-1 & Bp 668 & BURPS668_A2331-A2390 & 41.1 & 60 & । & prophage-like & & \\
\hline PI E264-1 (Gl1) & Bt E264 & BTH_I0091-I0119 & 49.1 & 26 & 1 & putative prophage & & \\
\hline PI E264-2 (Gl13) & Bt E264 & BTH_\|1325-II1368 & 33.1 & 41 & $\|$ & prophage-like & & \\
\hline PI E264-3 (Gl12) & Bt E264 & BTH_\|1011-||1070 & 52.0 & 62 & $\|$ & putative prophage & & \\
\hline PI LB400-1 & Bx LB400 & Bxe_A3036-A3110 & 53.4 & 40 & 1 & putative prophage & & \\
\hline PI CGD1-1 & Bmul CGD1 & BURMUCGD1_3398-3447 & 37.7 & 51 & 1 & putative prophage & & \\
\hline PI CGD1-2 & Bmul CGD1 & BURMUCGD1_2149-2203 & 45.6 & 56 & 1 & prophage-like & & \\
\hline PI CGD2-1 & Bmul CGD2 & BURMUCGD2_1176-1227 & 36.6 & 52 & । & putative prophage & & \\
\hline PI CGD2-2 & Bmul CGD2 & BURMUCGD2_2461-2520 & 44.6 & 60 & 1 & prophage-like & & \\
\hline PI CGD2-3 & Bmul CGD2 & BURMUCGD2_4590-4656 & 49.4 & 67 & $\|$ & prophage-like & & \\
\hline PI 17616-1 & $\begin{array}{l}\text { Bmul ATCC } \\
17616\end{array}$ & Bmul_1771-Bmul_1998 & 236.3 & 217 & । & putative prophage & & \\
\hline PI 17616-3 & $\begin{array}{l}\text { Bmul ATCC } \\
17616\end{array}$ & Bmul_3828-Bmul_3914 & 73.0 & 80 & $\|$ & prophage-like & & \\
\hline PI 17616-4 & $\begin{array}{l}\text { Bmul ATCC } \\
17616\end{array}$ & Bmul_4831-Bmul_4876 & 39.4 & 44 & $\|$ & prophage-like & & \\
\hline Gl3 (N/A) & Bp K96243 & putative prophage [3] & 51.2 & 31 & 1 & putative prophage & & \\
\hline Gl15 (N/A) & Bp K96243 & putative prophage [3] & 35.1 & 38 & $\|$ & putative prophage & & \\
\hline
\end{tabular}


Table 1 Sources and descriptions of bacteriophage and putative prophage islands (PI) used in this study. (Continued)

\begin{tabular}{|c|c|c|c|c|c|c|}
\hline \\
\hline \multicolumn{7}{|c|}{$\begin{array}{lll}\text { Description } & \text { Size } & \# \\
& (\mathrm{Mb}) & \text { ORFs }\end{array}$} \\
\hline \multicolumn{7}{|c|}{$\begin{array}{ll}\text { Ф1026b } & \text { Bp 1026b } \\
\text { (AY453853) } & \end{array}$} \\
\hline \multicolumn{7}{|l|}{ 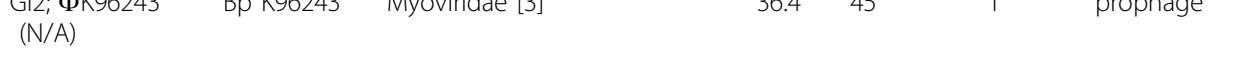 } \\
\hline \multicolumn{7}{|l|}{ (AF447491) } \\
\hline \multicolumn{7}{|l|}{ (AY539836) } \\
\hline $\begin{array}{l}\text { Bcep22 } \\
\text { (AY349011) }\end{array}$ & B. cepacia & Podoviridae & 63.9 & 81 & N/A & prophage \\
\hline $\begin{array}{l}\text { Bcep781 } \\
(\text { AF543311) } \\
\end{array}$ & B. cepacia & Myoviridae; [30] & 48.2 & 66 & $\mathrm{~N} / \mathrm{A}$ & prophage \\
\hline
\end{tabular}

\section{Negative staining}

To determine morphotypes, bacteriophages were prepared from $20 \mathrm{ml}$ of a plate culture lysate, incubated at $37^{\circ} \mathrm{C}$ for 15 min with Nuclease Mixture (Promega), precipitated with Phage Precipitant (Promega), and resuspended in 1 $\mathrm{ml}$ of Phage Buffer (Promega). The bacteriophage solution $(\sim 100 \mu \mathrm{l})$ was added to a strip of parafilm M (Sigma), and a formvar-coated nickel grid (400 mesh) was floated on the bacteriophage solution for $30 \mathrm{~min}$ at $25^{\circ} \mathrm{C}$. Excess fluid was removed, and the grid was placed on a drop of $1 \%$ phosphotungstic acid, $\mathrm{pH} 6.6$ (PTA) for $2 \mathrm{~min}$ at $25^{\circ} \mathrm{C}$. Excess fluid was removed, and the specimen was examined on a Philips CM100 transmission electron microscope. Nickel grids were glow discharged on the day of use.

\section{Bacteriophage sequencing and annotation}

Libraries were constructed from the genomic DNA from the bacteriophage isolates. Since the phage genomes were estimated to be $50 \mathrm{~kb}$ in size, sequencing, closure, and annotation was performed similar to that of a BAC sequence [23]. Each of the five isolated bacteriophages were completely sequenced to $10 \times$ coverage, closed, and annotated, and the sequences deposited in GenBank (Table 1A).

\section{Identification of putative prophages and prophage-like elements within strains}

Presence of prophage sequence within sequenced genomes of nine B. pseudomallei strains, six B. mallei strains [8], three B. multivorans strains, B. thailandensis E264 [24], and B. xenovorans LB400 [25] (Additional file 1, Table S2) was inferred using a number of similarity measures previously described [26,27]. First, the protein set of each genome was searched against a non-redundant database of viral proteins using BLASTP [28] with a cutoff of $\mathrm{e}^{-10}$. Secondly, the annotation of each strain was searched for several virus-related keywords such as integrase, tail, capsid, portal, terminase, etc. Clustering of such proteins with proteins containing similarity to known phage proteins as identified by BLASTP, as well as the orientation of proteins within clusters was considered strong evidence for prophage presence. Finally, tRNA genes and attachment sites were examined. A tRNA sequence immediately flanking an integrase was considered to be a potential att site, particularly if an exact repeat of least a $10 \mathrm{bp}$ of the tRNA was present within several thousand bp. Regions containing all of these factors (i.e., viral-like proteins clustered and in a specific orientation, and flanked by a tRNA/integrase on one end and an exact repeat of at least $10 \mathrm{bp}$ of the tRNA on the other end) were considered putative prophage. Regions containing many of these characteristics but lacking one or more, usually an integrase or repeat sequence, were considered prophage-like. Some att sites are less than $10 \mathrm{bp}$ and are difficult to spot so it is possible that some of the prophage-like elements may actually be true prophages. Prophage and prophage-like regions so inferred have been designated "PI-strain-1", "PIstrain-2", etc. (PI for Prophage Island), and are listed in Table 1B. Four of the B. pseudomallei strains represent two paired isolates from two separate patients, one strain isolated from an initial infection and the paired isolate from a re-emergent infection in the same patient.

Three of the 16 genomic islands previously identified in B. pseudomallei K96243 were included in the analysis, including $\varphi$ K96243 (GI2) and the putative prophages GI3 and GI15 [3]. Three prophage-like islands identified in B. thailandensis E264, GI1, GI12, and GI13, were also included in the analysis (Table 1B) [24]. Additionally, the published genome sequences of $\varphi 1026 \mathrm{~b}$ from $B$. pseudomallei 1026b [6], $\varphi \mathrm{E} 125$ from $B$. thailandensis E125 [21], BcepMu from B. cenocepacia J2315 [29], Bcep22 from B. cepacia, and Bcep781 from B. cepacia 
[30], all of which are classified as dsDNA phages, were included for comparison (Table 1C).

\section{Comparative genome sequence analysis and phylogenetic tree construction}

The program Dotter [31] was used to align nucleotide sequences of all isolated and putative prophage and prophage-like sequences and to identify initial groupings. To refine clusters, distance measures were calculated between all pairs of each of the 30 prophage and PI sequences. Reciprocal BLASTP comparisons of the translated protein sets were performed for each prophage/PI against all others. BLASTP distances between each pair were calculated according to the formula: 1-(number of significant hits between both genomes/total number of genes in both genomes) [32]. Distances were calculated using E value cutoffs of $1 \times \mathrm{E}^{-01}, 1 \times \mathrm{E}^{-05}$, and $1 \times \mathrm{E}^{-10}$. FITCH with the global and jumble options was used to generate a phylogenetic tree from each of the three distance matrices derived from the BLASTP distances [33].

Calculation of local collinear blocks (LCB or synteny blocks) was done using progressive Mauve alignment [34] with default settings. Initial identification of morons was conducted in the Mauve alignments by searching for ORFs that disrupted the collinearity in LCBs. Confirmation of morons was done by (i) comparing \% GC content of each ORF against the mean \% GC of phagespecific genes (i.e., involved in structure, replication, and host lysis); (ii) promoter and terminator prediction analysis with BPROM http://www.Softberry.com, PPP http://bioinformatics.biol.rug.nl/websoftware, PROMSCAN [35] or Promoter Prediction by Neural Network [36]; (iii) prediction of terminators with TransTermHP [37]; and (iv) search for homologs across different phage types within our data set and in the non-redundant database at NCBI.

\section{Phage gene expression analysis using RNAseq}

RNA from three biological replicates of $B$. pseudomalle $i$ DD503 (a derivative of 1026b) grown in LB was extracted from cells in early logarithmic growth using RNAeasy (QIAgen, Valencia CA). Ribosomal RNAs were removed by 2 rounds of MicrobExpress (Ambion, Foster City CA). Each RNA preparation was used in individual cDNA synthesis reactions using SuperScript II (Invitrogen, Carlsbad CA) and sequenced individually in the Illumina Genome Analyzer (Illumina Technologies, San Diego CA) or SOLiD instruments with 100 or $50 \mathrm{bp}$ reads, respectively. Data was analyzed using CLC Genomics Workbench allowing for 2 mismatches in each read and only one map location per read. Total gene expression was normalized according to the total number of reads in the library and the gene size, resulting in reads per kilobase per million reads (RPKM). Only genes that had more than 10 hits were considered to be expressed above the noise level.

\section{Results and Discussion}

\section{Isolated and sequenced bacteriophages}

Five bacteriophages were isolated from three $B$. pseudomallei and two B. thailandensis strains (Table 1A) when plaqued on B. mallei ATCC 23344 as a suitable host for bacteriophages $[3,6,21]$. Most B. pseudomallei and $B$. thailandensis strains only produced one phage, except for E12 and 644 which each produced at least two different phage particles. All of the bacteriophages contained long tails. Three were classified as P2-like viruses, one as a lambda-like virus, and one as a $\mathrm{Mu}$-like virus. The bacteriophage genomes ranged in size from 35.7 to $48.7 \mathrm{~Kb}$ and contained from 47 to 71 genes. Specific details about each of these bacteriophages are provided below, representative images of each isolated bacteriphage are shown in Fig. 1A and other properties are described in Table 1A.

$\varphi 52237$

B. pseudomallei Pasteur 52237 spontaneously produced a bacteriophage, designated $\varphi 52237$ that formed uniform, slightly turbid plaques on B. mallei ATCC 23344, suggesting that this strain produces only one bacteriophage under the growth conditions used. While it is plausible that different bacteriophages might form plaques with the same morphology, here we assumed that similar plaques were formed by only one bacteriophage. Based on its morphotype, $\varphi 52237$ can be classified as a member of the order Caudovirales and the family Myoviridae [38].

$\varphi$ E12-2

B. pseudomallei E12 spontaneously produced two bacteriophages, $\varphi E 12-1$ and $\varphi E 12-2$, that formed plaques on B. mallei ATCC 23344. $\varphi$ E12-1 produced turbid plaques of 0.5 to $1 \mathrm{~mm}$ in diameter and $\varphi \mathrm{E} 12-2$ produced turbid plaques with a diameter of 1.5 to $2.0 \mathrm{~mm}$. The purified plaques maintained their morphology following a further round of infection in the host suggesting that they were formed by two distinct bacteriophages. Approximately $10 \mathrm{pfu} / \mathrm{ml}$ of $\varphi \mathrm{E} 12-1$ and $\varphi \mathrm{E} 12-2$ were present in B. pseudomallei E12 culture supernatants. We were unable to isolate nucleic acid from $\varphi E 12-1$ and no further work was carried out on this bacteriophage. $\varphi$ E12-2 possessed an isometric head that was $\sim 62 \mathrm{~nm}$ in diameter and a contractile tail that was $\sim 152 \mathrm{~nm}$ long and $\sim 21 \mathrm{~nm}$ in diameter (Fig. 1A). Similar to p52237, $\varphi$ E12-2 can be classified as a member of the order Caudovirales and the family Myoviridae [38].

\section{ب644-2}

B. pseudomallei 644 spontaneously produced 2 bacteriophages, $\varphi 644-1$ and $\varphi 644-2$, that formed plaques on $B$. mallei ATCC 23344. $\varphi 644-1$ and $\varphi 644-2$ produced plaques of different size and turbidity. $\varphi 644-2$ was ten 
A

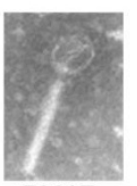

$\phi 52237$

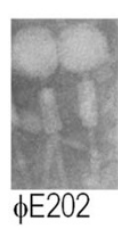

$\phi E 12-2$
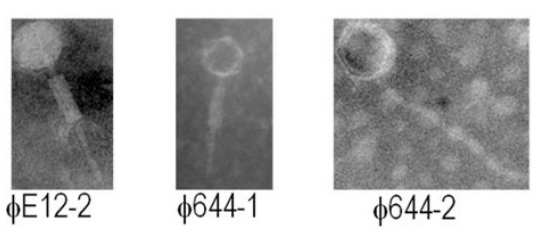

$\phi 644-2$

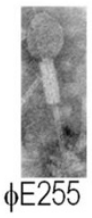

B

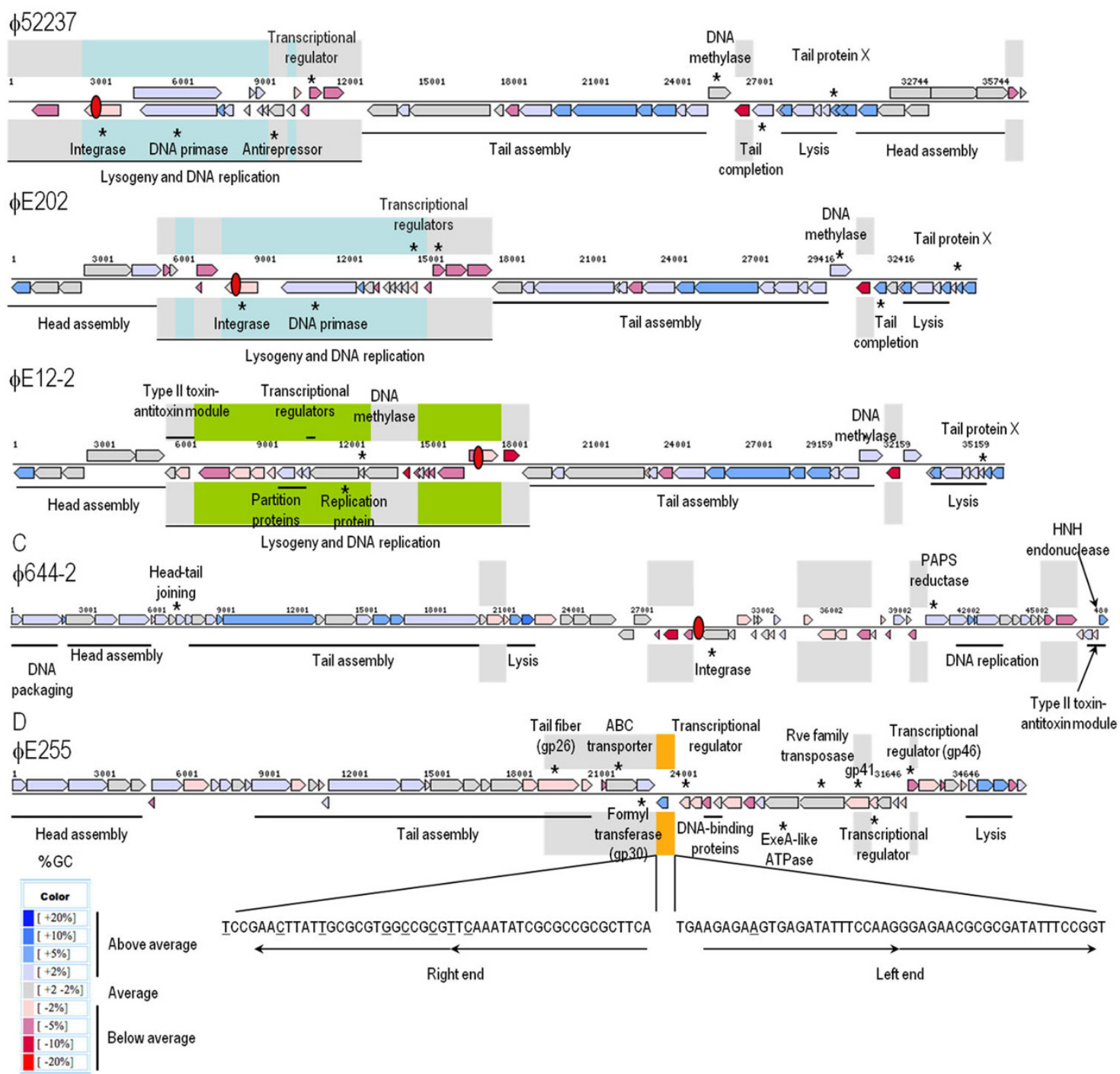

Figure 1 Transmission electron micrographs (TEM) of the Burkholderia bacteriophages analyzed in this project and schematic illustrations of their genomes. (A) TEM of bacteriophages negatively stained with 1\% phosphotungstic acid. (B) Schematic illustrations of the P2-like Myoviridae genomes of $\varphi 52237, \varphi E 202$, and $\varphi$ E12-2. Cyan shading represents sequences that are conserved in the subgroup A Myoviridae $\varphi 52237, \varphi E 202$, and $\varphi$ K96243 and lime shading represents sequences that are conserved in the subgroup B Myoviridae $\varphi$ E12-2, Gl15, and PI-E264-2. Gray shading represents sequences that are variably present in Myoviridae subgroups A and B. (C) Schematic illustration of the lambda-like Siphoviridae genome of $\varphi 644-2$. Gray shading represents sequences that are unique to $\varphi 644-2$. (D) Schematic illustration of the Mulike Myoviridae genome of $\varphi E 255$. Gray shading represents sequences that are unique to $\varphi \mathrm{E} 255$ and orange shading represents packaged host DNA. The 23-bp imperfect direct repeats at the left and right ends of the $\varphi E 255$ genome are shown and sequence differences with the repeat sequences of BcepMu are underlined. Genomic illustrations were obtained from the Integrated Microbial Genomes website http://img.jgi.doe. gov/cgi-bin/pub/main.cgi. Genes are shown as arrows that are pointing in their relative direction of transcription and are color coded based on their \% GC composition (see scale at bottom). Individual genes with functional annotations are labeled and designated with an asterisk (*) while groups of genes with a common function are labeled and designated with a line. The locations of att sites are shown as red oblong circles. Nucleotide sequence numbering is shown above each genome. 
times more abundant in B. pseudomallei 644 culture supernatants. Based on its morphology, $\varphi 644-2$ can be classified as a member of the order Caudovirales and the family Siphoviridae [38]. The genome of $\varphi 644-1$, a member of the Myoviridae family, could not be determined in this study.

\section{$\varphi$ E255}

B. thailandensis E255 spontaneously produced a bacteriophage, designated $\varphi \mathrm{E} 255$, which formed turbid plaques with a diameter of $\sim 0.5 \mathrm{~mm}$ on B. mallei ATCC 23344 . No other plaque types were identified. Based on its morphotype, $\varphi$ E255 can be classified as a member of the order Caudovirales and the family Myoviridae [38].

$\varphi$ E202

B. thailandensis E202 spontaneously produced a bacteriophage, designated $\varphi \mathrm{E} 202$, which formed turbid plaques on B. mallei ATCC 23344. No other plaque types were identified. $\varphi$ E202 production was increased 55 -fold by brief exposure to UV light (data not shown). Based on its morphotype (Fig. 1A), $\varphi$ E202 can be classified as a member of the order Caudovirales and the family Myoviridae [38]. We examined the host range of $\varphi$ E202 using 17 Burkholderia species (Additional file 1, Table S1). Bacteriophage $\varphi \mathrm{E} 202$ formed plaques on 9 of 10 natural $B$. mallei strains. It also formed plaques on a capsule-deficient mutant derived from ATCC 23344, DD3008 [39], suggesting that the capsular polysaccharide is not required for $\varphi \mathrm{E} 202$ attachment. In contrast, two $B$. mallei strains that do not produce lipopolysaccharide (LPS) were resistant to plaque formation by $\varphi$ E202; NCTC 120 and DD110795 (a laboratory-passaged derivative of ATCC 15310), which suggests that LPS is a receptor, or co-receptor, for $\varphi \mathrm{E} 202$. Given the $>90 \%$ nucleotide identity of the tail assembly genes of the Burkholderia Myoviridae, it is likely that they all share the same receptor(s). Unlike other characterized Burkholderia Myoviridae ( $\varphi$ E125, $\varphi 1026 b), \varphi E 202$ forms plaques on a species other than B. mallei (Additional file 1, Table S1), namely 3 strains of B. pseudomallei; NCTC 4845, STW 199-2, and STW 115-2. It is currently unknown why these $B$. pseudomallei strains exhibit plaque formation with $\varphi$ E202 while others do not. No other Burkholderia species examined formed plaques with $\varphi$ E202 (Additional file 1, Table S1).

\section{Genomic analysis of the Burkholderia phages I. Myoviridae subgroup $A$ and $B$}

Based on sequence similarity, $\varphi 52237, \varphi E 202$, and $\varphi$ K96243 belong to subgroup A of the Myoviridae and $\varphi$ E12-2, GI15, and PI-E264-2 to subgroup B (Fig. 2). Furthermore, the genomic structure of each of these are arranged in multigene "modules" that encode proteins involved in a common function, such as DNA packaging, head biosynthesis, tail biosynthesis, host lysis, lysogeny or DNA replication [40,41] (Fig. 1B). The relative order of these modules in $\varphi 52237, \varphi \mathrm{E} 202$, and $\varphi \mathrm{E} 12-2$ is similar to that of bacteriophages $\mathrm{P} 2$ and $\varphi$ K96243 [42]. The order is also conserved in bioinformatically-identified prophage-like elements GI15 of $B$. pseudomallei K96243 and PI-E264-2 of B. thailandensis E264 (see below).

The modules for tail assembly, lysis, and head assembly of all Myoviridiae phages were highly conserved (Fig. 1B). However, the region encoding for lysogeny and DNA replication contained abundant genetic variability. Some of the genes within this region were present exclusively in either of the subgroups (Fig. 1B). This suggests that the mechanism of integration, regulation of excision, and/or replication of episomal bacteriophage DNA could be distinct for subgroup A and B Myoviridae. For example, subgroup A bacteriophage genomes encode DNA primase proteins which catalyze the synthesis of short RNA primers required for DNA replication by DNA polymerases (Fig. 1 B). Subgroup B bacteriophages, on the other hand, encode for ParA-like partitioning proteins which are ATPases involved in chromosome partitioning. In addition, subgroup B genomes encode replication gene A protein-like sequences. Members of this family of proteins are endonucleases which introduce single-strand nicks at or near the origin of replication (Fig. 1B).

Among the conserved regions, some segments are variably present in the bacteriophages and PIs (Fig. 1B). It is likely that these regions were acquired by recombination with unrelated bacteriophages (or prophages), and that these segments might be considered 'morons' [20]. This is supported by the fact that these regions exhibit a lower \% GC content relative to the rest of the bacteriophage genomes (Fig. 1B), which suggests horizontal transfer of genetic information. Most of these novel genes encode conserved hypothetical proteins which have no defined functional activities, but share similarity with proteins in other bacteriophages. No obvious virulence factor genes are encoded by these bacteriophage genomes, which is consistent with a previous report on this topic [42]. Interestingly, $\varphi$ E12-2 gp6 and gp7 appear to encode a type II toxin-antitoxin (TA) addiction module (Fig. 1B - see below) [43]. Other novel proteins are encoded by the $\varphi 52237, \varphi \mathrm{E} 202$, and $\varphi \mathrm{E} 12-2$ genomes (Fig. 1B), but no functions can be assigned to these gene products at this time.

The phage attachment sites (attP) of $\varphi 52237$ and $\varphi$ E202 are found at the $3^{\prime}$ ends of putative site-specific integrase genes (Fig. 1B) and are identical to each other. The nucleotide sequence of attP contained a 45-bp sequence that was identical to the $3^{\prime}$ end of the phenylalanine tRNA (GAA) gene on chromosome 1 of $B$. pseudomallei $\mathrm{K} 96243$ (positions 145,379-145,454). This attP 


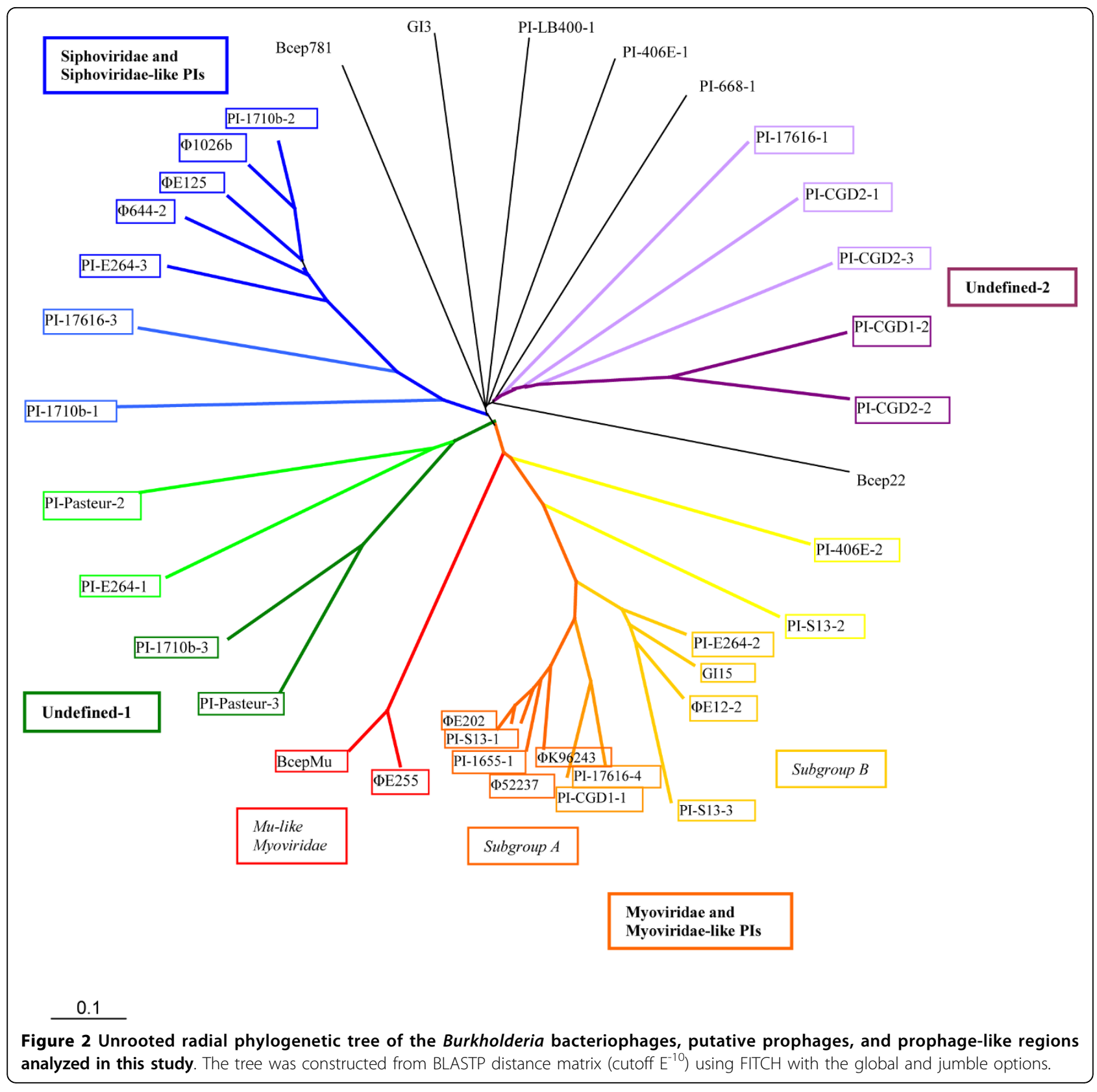

site is also utilized by $\varphi$ K96243 [3]. The integrase genes of these three subgroup A Myoviridae terminate with the tRNA (Phe) gene when integrated as prophages, but not when the bacteriophage genomes are episomal. Thus, following integration the integrase gene is partitioned into two fragments.

The $\varphi E 12-2$ attP site is located between gp24 and gp25 (5'-AATTTGACATAAGGTAAA-3') (Fig. 1B) and is identical to the sequence at both ends of GI15 in $B$. pseudomallei $\mathrm{K} 96243$ [3]. This integration site is present in an intergenic region on the $B$. pseudomallei genome and does not disrupt any obvious ORFs. This attP site does not have any homology to tRNAs. PI-E264-2 is also flanked by a similar sequence (5'-ATTTGACATAACGTAAA-3') in B. thailandensis E264, suggesting that it also uses this attP. No obvious integrase genes are encoded by $\varphi$ E12-2, GI15, or PI-E264-2, which suggests these subgroup B Myoviridae use a different mechanism of integration.

\section{Mu-like phages}

The $\varphi E 255$ genome shares 90\% nucleotide sequence identity with the genome of BcepMu, a Mu-like bacteriophage spontaneously produced by Burkholderia cenocepacia strain J2315 [29]. Similar to BcepMu, the $\varphi$ E255 
genome can be divided into functional clusters from the left end to the right end of the linear phage genome: replication and regulation, host lysis, head assembly, and tail assembly (Fig. 1D). $\varphi$ E255 encodes a transposase with a Rve integrase domain (gp40, PFAM PF00665) that allows transposition as a mechanism of replication. Following replicative transposition, DNA is packaged into the bacteriophage heads using a pac site at the left end of the bacteriophage genome which allows 2002,000 bp of flanking host DNA to also be packaged [29]. The genomic sequence of $\varphi$ E255 (accession number NC_009237) contains 467 bp of host DNA sequence (Bm ATCC23344). The left and right ends of the linear $\varphi$ E255 genome contain 23-bp imperfect direct repeats that could be recognized by gp40 during replicative transposition (Fig. 1D). These repeats are similar to those found at the ends of the BcepMu genome [29] and the nucleotide differences are underlined in Fig. 1D.

Three regions of the $\varphi \mathrm{E} 255$ genome are not present in the BcepMu genome and appear to be $\varphi \mathrm{E} 255$-specific (gray shading in Fig. 1D). The unique regions are found at the left and right ends of the $\varphi \mathrm{E} 255$ genome, which is consistent with the location of unique sequences in BcepMu and other BcepMu-like prophages [29]. The two unique genes on the left side of the bacteriophage genome, gene 41 and gene46, encode a conserved hypothetical protein and a lambda $C 1$ repressor-like transcriptional regulator, respectively (Fig. 1D). These proteins are presumably involved in $\varphi$ E255 activation and/or replication. Five unique genes are encoded on the extreme right end of the $\varphi$ E255 genome, including genes 26-30 (Fig. 1D). Gp26 encodes a putative tail fiber protein which presumably is required for attachment and probably provides host receptor specificity to this bacteriophage. It is interesting that this gene, and the downstream tail assembly chaperone protein (gp27), are the only tail assembly genes that are not conserved in BcepMu. This suggests that the BcepMu receptor(s) on B. cenocepacia is distinct from the $\varphi \mathrm{E} 255$ receptor(s) on B. thailandensis and B. mallei. Furthermore, it suggests that the unique tail fiber protein and a tail assembly chaperone protein (gp27) were either acquired by $\varphi$ E255 via horizontal transfer or lost by BcepMu. Gp28 is a hypothetical protein with no functional prediction, but gp29 is a putative ABC (ATP-binding cassette) transporter protein (Fig. 1D). It is possible that $\varphi \mathrm{E} 255$ gp29 is involved in the import of a nutrient or export of toxic metabolites that confers a selective advantage on the lysogen harboring it. On the other hand, Lactococcus lactis bacteriophage P335 [44] and Streptococcus pyogenes bacteriophage $\varphi \mathrm{NIH} 1.1$ [45] also encode ABC transporters and these molecules may play an undefined role in the bacteriophage lifecycle. Finally, gp30 is a putative formyl transferase domain protein (Fig. 1D), a family of proteins involved in a variety of biochemical pathways, including de novo purine biosynthesis, methionyl-tRNA biosynthesis, and formate biosynthesis. None of these $\varphi \mathrm{E} 255$ genes have homologs in any of the other phage/PI or Burkholderia genomes reported here or elsewhere.

\section{Siphoviridae}

The gene order and modular organization of the $\varphi 644-2$ genome is reminiscent of lambdoid bacteriophages, including $\varphi 1026 \mathrm{~b}$ and $\varphi \mathrm{E} 125[6,21,46,47]$. The $\varphi 644-2$ genome harbors five regions that are specific to $\varphi 644-2$ and contain a lower GC content than the rest of the (644-2 genome, suggesting they may have been acquired horizontally from a novel source (gray shading in Fig. 1C). The thirteen novel genes present in these regions encode hypothetical proteins with no known function (gp22, gp23, gp24, gp33, gp34, gp35, gp46, gp47, gp48, gp49, gp55, gp66, and gp67). The genome also contains several interesting features, including a putative phosphoadenosine phosphosulphate (PAPS) reductase (gp56), a putative type II toxin-antitoxin module (gp69 and gp70), and a putative $\mathrm{HNH}$ endonuclease (gp71) that might be advantageous to the phage or its lysogen (Fig. 1C; discussed further below).

The $\varphi 644-2$ genome contains ten base $3^{\prime}$ singlestranded extensions on the left ( $3^{\prime}$-GCGGGCGAAG-5') and right (5'-CGCCCGCTTC-3') (Fig. 1C). In $\varphi$ E125, this sequence serves as a cohesive $(\cos )$ site [21], suggesting that $\varphi 644-2$ uses the same cos site as $\varphi E 125$. The nucleotide sequence immediately downstream of gene36, which encodes a putative site-specific integrase, contained the candidate attP site of $\varphi 644-2$. It is characterized by a 30 -bp sequence that was identical to the 3 ' end of a 90-bp serine tRNA (GGA) gene on the B. pseudomallei K96243 small chromosome [3,4] (Fig. 1C). Interestingly, a 19-kb prophage-like island (GI13) is also integrated at this location in the B. pseudomalle K96243 genome [3,4], although there is no sequence similarity between the two elements.

\section{Inferred prophage islands}

Twenty-four putative prophage or prophage-like regions were identified in 11 of the 20 Burkholderia strains (Table 1B). In addition, two GIs from K96243 (GI3 and GI15) were included in subsequent analysis since these also classify as putative prophage by our definition [3]. We call these regions prophage islands (PI) defined as regions of the genome that were found to contain most if not all of the elements characteristic of prophages (see Materials and Methods), but have not been isolated and experimentally characterized. Most B. pseudomallei and all $B$. multivorans strains were found to contain PIs; three were identified in $B$. thailandensis E264, one in $B$. xenovorans LB400, and none in any of the B. mallei 
strains. The three B. thailandensis E264 PIs, PI-E264-1, -2 , and -3 , correspond to B. thailandensis GI1, Bt GI13, and Bt GI12, respectively, as described by $\mathrm{Yu}$ et al [24], although the range of genes in the PIs described here differ slightly due to our criteria for inclusion. Similarly, PI-668-1 corresponds to GI15c from B. pseudomallei MSHR668 in Tuanyok et al [4]. As mentioned above, no PIs were detected in $B$. pseudomallei $1106 \mathrm{a} / \mathrm{b}$, although phage-like remnants were found in these strains. Overall, seventeen of the 24 identified regions were located on chromosome I of the respective bacterial strain, and all but five were putative prophages (i.e., most likely to be active prophages containing all of the prophage-like elements described in the Materials and Methods). Of the seven regions located on chromosome II, one (PIE264-3) was classified as a putative prophage, while the remaining six were designated prophage-like.

\section{Paired strains B. pseudomallei $1710 \mathrm{a} / \mathrm{b}$ and B. pseudomallei 1106a/b}

The two pairs B. pseudomallei $1710 \mathrm{a} / \mathrm{b}$ and B. pseudomallei $1106 \mathrm{a} / \mathrm{b}$ represent two bacterial strains isolated at different time points from the same two infected patients, isolated from the primary infection (a) and the relapse (b). We hypothesized that difference/s in sequence relating to the relapse or host selection would be detected, either in the form of SNPs/indels or as variation in the phage harbored within each strain. Three PIs were identified in each of the B. pseudomallei 1710 strains. PI-1710a/b-1 is immediately followed by PI$1710 \mathrm{a} / \mathrm{b}-2$ on chromosome I, separated by a tRNA pseudogene in each strain. This region is described as GI6b in Tuanyok et al. [4]. PI-1710a/b-3 is located further downstream on chromosome I. All three regions are nearly identical, averaging 98.4, 97.7, and 96.6\% identity over 98.2, 97.1, and $96.2 \%$ of length (for $-1,-2$, and -3 , respectively). PI-1710a-1 and PI-1710b-1 are 41.3 and $41.4 \mathrm{~kb}$ in length, respectively, and both are bounded by tRNA-Pseudo- 2 and a 23 bp exact repeat of the $3^{\prime}$ end of this tRNA. Both PI-1710a-2 and PI-1710b-2 are 60.6 $\mathrm{kb}$ in size and are bounded by tRNA-Pro- 2 and a $49 \mathrm{bp}$ exact repeat. The prophage-like regions in both strains (PI-1710a-3, PI-1710b-3) are defined by the presence of a phage integrase at the $3^{\prime}$ end by tRNA-Thr-3, with several viral-like proteins immediately upstream, but no repeat region could be identified to define the $5^{\prime}$ end. Both are $62.8 \mathrm{~kb}$. Since the three prophage islands are nearly identical between $B$. pseudomallei 1710a and $B$. pseudomallei $1710 \mathrm{~b}$, from here on we will only refer to B. pseudomallei $1710 \mathrm{~b}$ and associated prophage islands. These results indicate that the prophage in $\mathrm{Bp} 1710 \mathrm{a} / \mathrm{b}$ were not excised and did not experience any significant changes even after passage through a host.
By the definitions set forth for prophage islands given in this work, no PIs were identified in either of the $B$. pseudomallei 1106 strains. Tuanyok et al. [4] described 16 genomic islands in 1106a, one of which was identified as a putative prophage (GI10.2; BURPS1106A_3666 - 3701). However, this region also contains three transposases, and so was not considered in the analysis reported here.

\section{Bacteriophage clusters}

Results from the Dotter analysis allowed a preliminary clustering of prophages and prophage-like regions. These groups were further refined by examination of BLASTP protein distance data, resulting in the clustering of 32 of the 37 PIs and prophages into each of four groups (data not shown). Cluster composition was very similar between the three BLASTP-distance FITCH trees and agreed with DOTTER results, although branch positions varied slightly (Fig. 2). Seven prophages/PIs clustered into the Siphoviridaelike group, so named because of the inclusion of the previously published bacteriophages $\varphi 1026 \mathrm{~b}$ [6] and $\varphi$ E125 [21]. Bacteriophage $\varphi 644-2$, described in this study, is also a member of this group (Fig. 2). Prophages in this group have long non-contractile tails and termini with cohesive ends. The cos site, present in $\varphi 1026 \mathrm{~b}$ and $\varphi \mathrm{E} 125$, was identified in all other members of this group.

The Myoviridae-like group consists of 15 prophages/ PIs (Fig. 2). Phages in this group, identified by the inclusion of $\varphi$ K96243 (GI2) [3] and $\varphi 52237$, typically have contractile tails and terminal repeats [48]. Three subgroups were identified within the Myoviridae-like class (Fig. 2). Subgroup A contains $\varphi$ K96243, $\varphi 52237, \varphi$ E202, and four other prophages/PIs. Bacteriophage $\varphi E 12-2$ and five prophages/PIs clustered to form subgroup B, including two (PI-406E-2 and PI-S13-2) which appear to be more distantly related. The $\mathrm{Mu}$-like Myoviridae group contains only two prophages: BcepMu [29] and $\varphi \mathrm{E} 255$. Both left and right phage ends at the host/phage junction in BcepMu [29] were located at the ends of $\varphi$ E255, with $95 \%$ and 91\% identity, respectively. No significant identity was found between either of the two $\mathrm{Mu}$-like prophages and any of the other prophages or prophage-like sequences.

Two undefined groups were also identified: undefined1 contains four PIs, and undefined-2 has five (Fig.2). Interestingly, undefined-2 contains five of the eight PIs identified in the three B. multivorans strains. Finally, six sequences had no significant similarity to any other sequence and were thus considered unclustered, including PI-668-1, PI-406E-1, PI-LB400-1, GI3, Bcep22 and Bcep781. 


\section{Burkholderia bacteriophages are populated by morons} Genomic comparisons of all the phages in each class revealed that the genomes are arranged in mosaic structures. Each of the phylogenetic classes of phages contains distinct local collinear blocks (LCB), also called synteny blocks, which are differentially present among the phages in that group (Fig. 3). Within each group, the synteny blocks are shuffled among the genomes (Fig. 3), suggesting that several of the phages have undergone dramatic genomic rearrangements. In several instances, synteny blocks

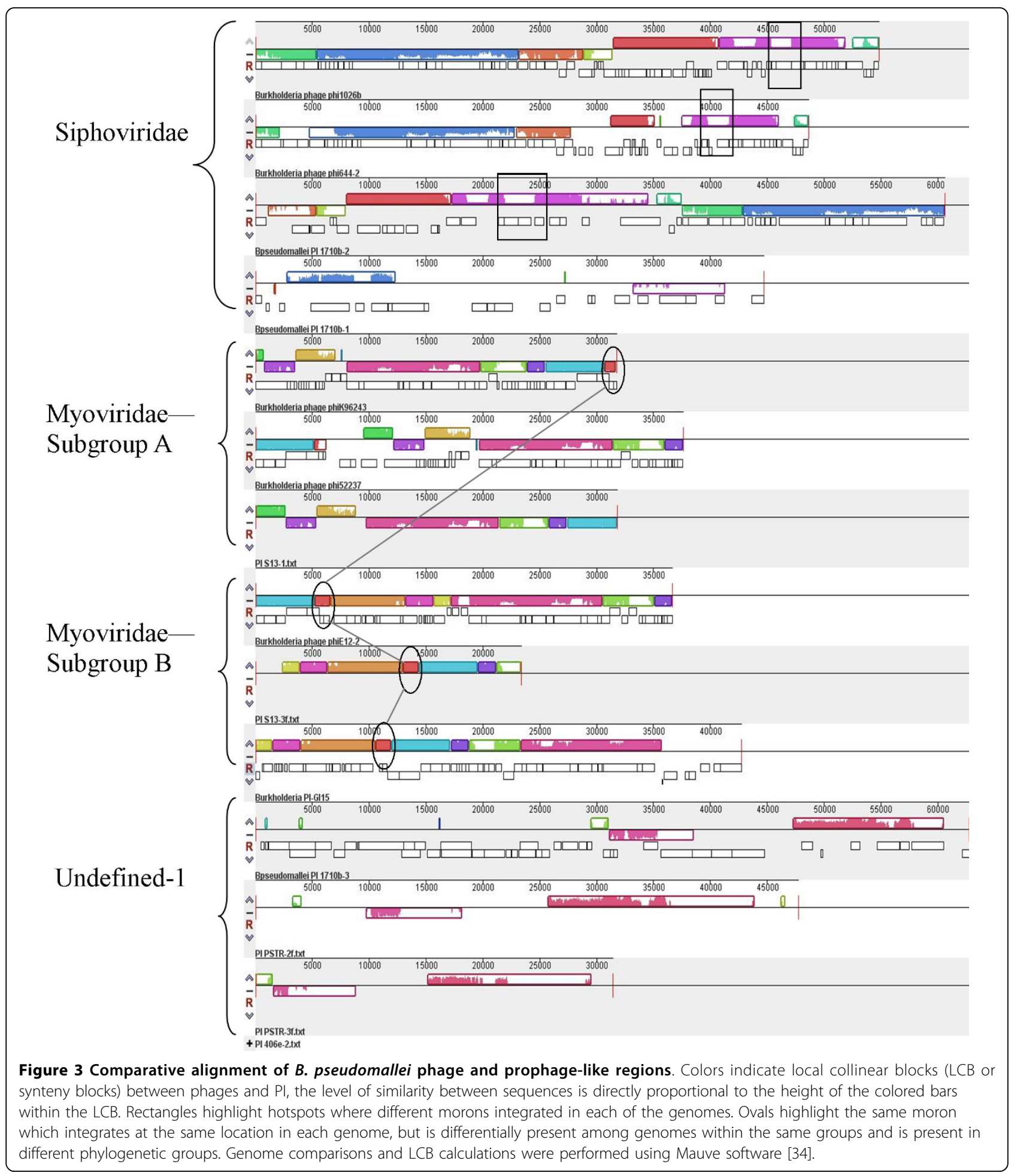


were either abruptly cut off at one end, or a predicted ORF appeared within the block in one genome, but was absent in other phages (see white areas within LCB in Fig. 3). We hypothesized that these randomly occurring ORFs could be morons, i.e. genetic elements that integrate between adjacent phage genes, which may confer some additional fitness to the bacteriophage [20]. Aside from being inserted among phage genes, morons usually (i) have their own transcriptional control system (a promoter, operator, and/or terminator), (ii) have a different GC content than the rest of the phage, and (iii) may be found in more than one class of phage [20].

In most cases, the randomly occurring ORFs detected by LCB analysis of the Burkholderia phages displayed at least 2 of the characteristics of morons (data not shown), and thus we classified them as morons themselves. In total, 17 different morons were identified among the phages and prophages-like islands (Table 2). Some of the PI/phages had as many as 12 morons, while some had as few as 2 (Table 2). Among different phage genomes, morons appeared adjacent to homologous genes across phylogenetic groups (see ovals in Fig. 3), and in many cases, some regions seemed to be hotspots for moron entry since different morons were detected at the same location (see below; Fig. 4).

Analysis of predicted functions of the Burkholderia morons shows that several of these proteins may enhance bacteriophage fitness, and thus replication, as proposed for other morons [20]. For example, two different morons containing toxin-antitoxin modules were found among the Myoviridae and Siphoviridae groups (Table 2). Interestingly, the T-A module in the Myoviridae phages is similar to two modules present in other $B$. pseudomallei and even B. mallei strains in regions containing phage remnants (data not shown), suggesting that this moron can persist even after the phage has been excised from the genome.

Several of the morons appeared to enhance the metabolic versatility of the host: aromatic compound degradation, iron transport and acquisition, and sulfate assimilation (Table 2). For example, it has been suggested that the PAPS reductase gene, which functions in the assimilatory sulfate reduction pathway, could serve as a fitness factor under conditions of iron limitation for the lysogens that harbor prophages encoding this enzyme [42]. PAPS reductase genes were identified in three members of the Siphoviridae-like group, $\varphi$ E125, $\varphi 644-2$ and PI-E264-3 (Fig. 4), and in the Myoviridae-like B subgroup member PI-E264-2. The PAPS reductase moron incorporated between two highly conserved phage genes (Fig. 4) at a location that appears to be an insertion hotspot, since the other members of this group contain different morons (Fig. 4 and rectangles in Fig. 3).
Other morons appear to be associated with enhanced host or bacteriophage competitiveness. For example, morons within the Myoviridae, Undefined-1, Undefined2, and Siphoviridae encode for the production of toxins that inhibit the growth of competing bacterial strains (bacteriocins) and/or their associated translocation mechanisms (Table 2). Other morons could prevent infection of their host by other phage, these include morons that encode for site-specific endonucleases, DNA methylases, restriction-modification systems, phage abortive infection resistance, and phage-growth limiting genes. Although we could not confirm that GI3 from K96243 contains morons (since LCB analysis was limited to those PIs that formed clusters), two separate reverse-transcriptase (RT) modules are encoded in this PI. Many phage-encoded RT described to date also function in phage resistance by directly targeting other phage DNA.

Lastly, some of the morons encode for proteins associated with bacterial virulence (Table 2). Two different morons encode patatin-like phospholipases (PTP), which in $P$. aeruginosa can act as cytotoxins necessary for virulence in amoeba and contribute to lung injury in a mouse model $[18,49,50]$. Moreover, a prophageencoded phosholipase in group A Streptococcus also appears to enhance virulence and its expression results in more severe disease [49]. Two other morons encode for a proteophosphoglycan and a lytic transglycosylase, both of which have been associated with virulence in other pathogens [51]. Thus, some phages in Burkholderia spp. might also be implicated in enhanced virulence.

\section{Moron and phage genes are differentially expressed in Bp DD503}

We performed transcription analysis using RNAseq to determine to what extent phage genes and morons are expressed in $\varphi 1026 \mathrm{~b}$. The results demonstrate that most phage genes are normally not expressed in rich laboratory growth conditions (Table 3 ), and allowed us to determine at least one putative repressor that maintains such regulation. For $\varphi 1026 \mathrm{~b}$, the candidate repressor gene (phi1026bp79) had a very high expression value which was 4-times higher than any of the phage structural or replication genes, (Table 3). The protein (gp78) contains a divergent AAA domain involved in ATPbinding and has similarities to other proteins annotated as transcriptional factors, such as YP_002500546 from Methylobacterium nodulans ORS 2060. Most of the phage morphogenesis and replication genes are only expressed at low levels, with many genes (54 of 89 genes) not having any detectable expression (Table 3 ). In many phages, gene expression and lysogenic conversion occur only when the levels of the repressor protein 
Table 2 Morons identified among phages and PI in Burkholderia species.

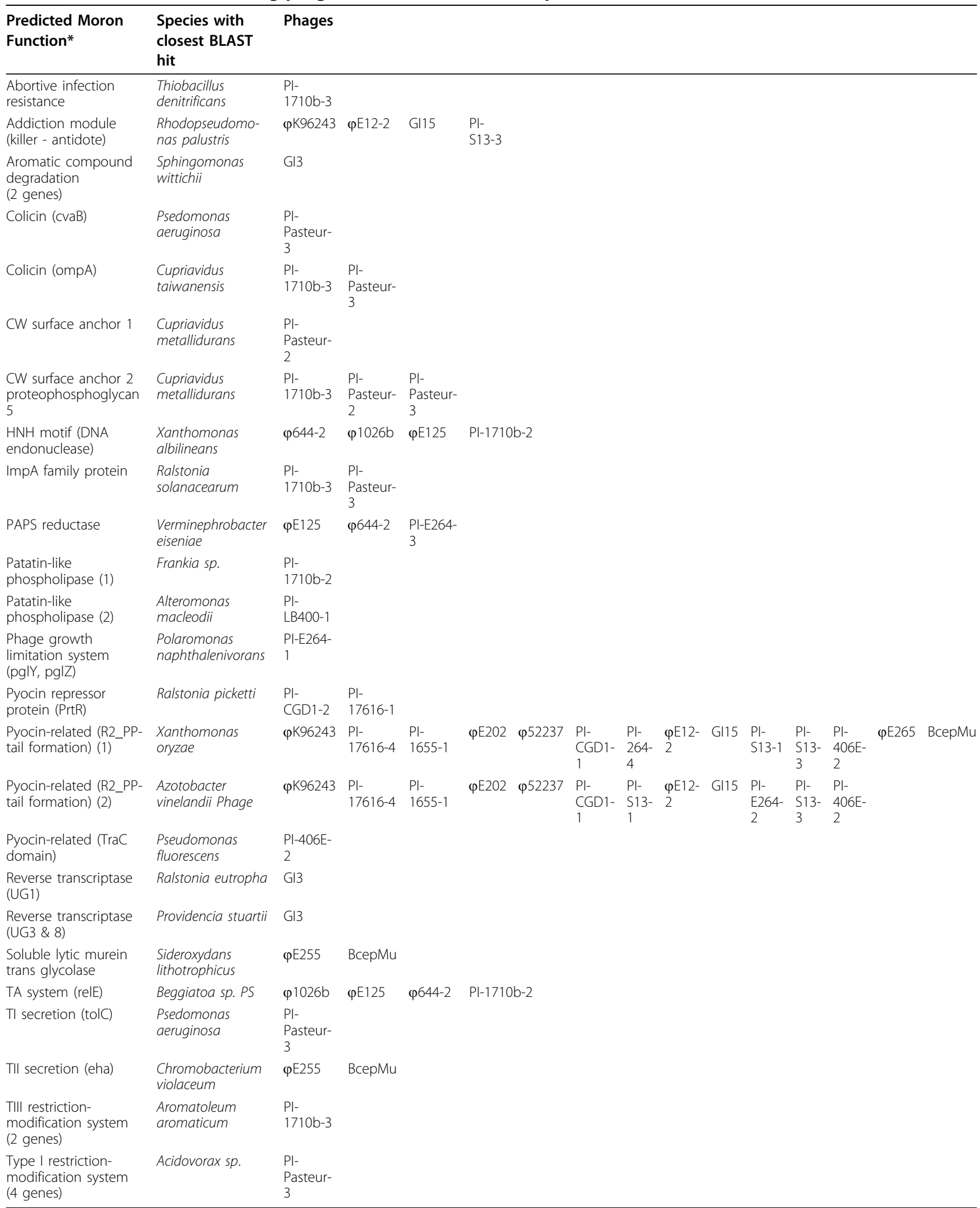

*Morons were identified as described in Methods. Phages listed in each column contain the predicted moron function. Non-Burkholderia species that have the closest protein as identified by BLASTp (E value less than $10^{-3}$ ) are presented. 


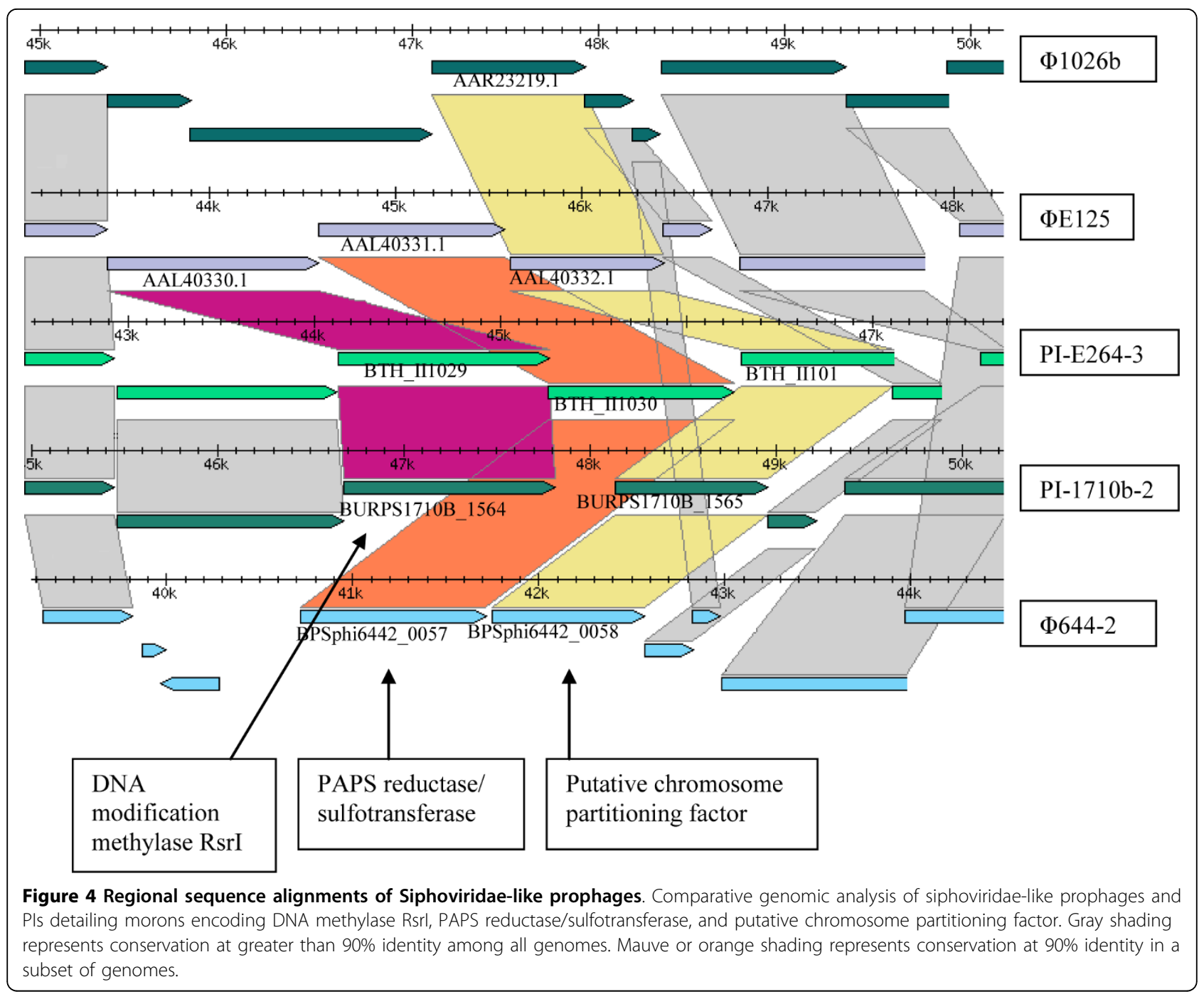

drop below a certain threshold. None of the other phages identified in this study had proteins with homology to this putative repressor suggesting that their mechanisms of regulation are different.

In addition to the highly expressed repressor, several of the morons in $\varphi 1026 \mathrm{~b}$ were also expressed, consistent with the notion that morons are differentially regulated from the rest of the prophage genes as proposed by Hendrix et al [20]. The toxin-antidote morons were highly expressed, with the toxin gene (phi1026bp82) 1.5-fold higher than the antidote gene (phi1026bp81; Table 3). The DNA methylase and restriction modification moron (phi1026bp28-30), major facilitator permease, LysR transcriptional regulator and other morons with hypothetical proteins present among the genome were also highly expressed (Table 3) independently of the phage genes in their vicinity, further suggesting that phages represent a rich source of fitness factors that benefit the host even while the phage genes are repressed.

\section{Conclusions}

The vast diversity in pathogenicity, clinical presentation, and living environments that exists within and between the Burkholderiae can be attributed at least in part to the presence of prophages and prophage-like elements within the genomes of these microbes. In this report we have characterized and classified 37 prophages, putative prophages, and prophage-like elements identified from several Burkholderia species and strains within species. Five spontaneously produced bacteriophages of lysogenic B. pseudomallei and $B$. thailandensis were isolated and characterized, including their host range, genome structure, and gene content. Using bioinformatic techniques, 24 putative prophages and prophage-like elements were identified within whole genome sequences of various Burkholderia species. Interestingly, while putative prophages were found in all but one of the B. pseudomallei strains none were detected in any of the $B$. mallei 
Table 3 RNASeq analysis of gene expression of phage genes in Bp DD503.

\begin{tabular}{|c|c|c|}
\hline Gene & Annotation & Expression value (RPKM)* \\
\hline phi1026bp03 & putative portal protein & 3,601 \\
\hline phi1026bp05 & putative major capsid protein & 4,743 \\
\hline phi1026bp14 & putative tail length tape measure protein & 1,038 \\
\hline phi1026bp16 & hypothetical protein & 3,986 \\
\hline phi1026bp27 & putative DNA adenine methylase & 21,563 \\
\hline phi1026bp28 & hypothetical protein & 199,000 \\
\hline phi1026bp29 & PAAR repeat-containing protein & 186,000 \\
\hline phi1026bp30 & VRR-NUC domain protein & 132,500 \\
\hline phi1026bp31 & hypothetical protein & 77,624 \\
\hline phi1026bp32 & hypothetical protein & 8,751 \\
\hline phi1026bp33 & hypothetical protein & 17,084 \\
\hline phi1026bp34 & putative site-specific integrase & 5,746 \\
\hline phi1026bp36 & hypothetical protein & 23,220 \\
\hline phi1026bp37 & hypothetical protein & 80,994 \\
\hline phi1026bp38 & hypothetical protein & 16,224 \\
\hline phi1026bp44 & hypothetical protein & 2,494 \\
\hline phi1026bp48 & hypothetical protein & 2,501 \\
\hline phi1026bp51 & hypothetical protein & 26,846 \\
\hline phi1026bp59 & putative LysR family transcriptional regulator & 18,809 \\
\hline phi1026bp60 & putative major facilitator family permease & 29,669 \\
\hline phi1026bp61 & hypothetical protein & 33,472 \\
\hline phi1026bp62 & hypothetical protein & 46,783 \\
\hline phi1026bp63 & hypothetical protein & 10,273 \\
\hline phi1026bp64 & hypothetical protein & 219,500 \\
\hline phi1026bp65 & hypothetical protein & 220,000 \\
\hline phi1026bp78 & hypothetical protein & 4,184 \\
\hline phi1026bp 79** & putative transcriptional regulator & 59,976 \\
\hline phi1026bp81 & XRE familiy putative transcriptional regulator & 53,561 \\
\hline phi1026bp82 & addiction module toxin, RelE/StbE family & 92,307 \\
\hline
\end{tabular}

*Genes in bold belong to morons. Only genes with 10 or more reads are displayed, genes with fewer than 10 reads are considered non-expressed since they are not above noise level. Expression values are measured as reads per kilobase of coding sequence per million reads (RPKM). Number of reads and expression values are from one Illumina run, but are representative of 3 runs.

${ }^{*}$ Candidate phage repressor.

strains searched. The $B$. mallei genome is nearly identical to that of $B$. pseudomallei, differing by several contiguous gene clusters in B. pseudomallei that appear to have been deleted from $B$. mallei, and it is hypothesized that $B$. mallei evolved from a single $B$. pseudomallei strain $[8,9]$. If true, it is likely that this $B$. pseudomallei strain had at least one prophage within its genome that was excised from $B$. mallei leaving behind a toxin-antitoxin module. The prophage excision was part of a major host adaptation in $B$. mallei that also removed $\sim 1200$ other genes [8]. In addition, B. mallei is largely confined to a mammalian host in nature and is less likely to be exposed to new bacteriophages in this niche relative to other Burkholderia species that are commonly found in the soil/plant rhizosphere. Taken together, prophage elimination and limited prophage acquisition probably account for the lack of functional prophages in the $B$. mallei genome.
Sequences of the five isolated and sequenced bacteriophages, the 24 inferred prophages, and eight previously published Burkholderia prophages or putative prophages were classified based on nucleotide and protein sequence similarity, and an unrooted radial tree was constructed to estimate genetic relatedness between them. Several sequences could be classified as Siphoviridae-like, Myoviridae-like, or Mu-like Myoviridae based on similarity to phages known to be members of these groups. Additionally, two novel groups were detected, and five prophages/PIs could not be grouped with other phages. For the most part the phage groups were represented across all species and strains, with the notable exception of the undefined-2 group, which is composed primarily of $B$. multivorans-derived PIs (five from $B$. multivorans, one from $B$. pseudomallei), albeit loosely related. Further work that includes prophages derived from environmental and clinical isolates from other 
Burkholderia species as well as from other microbes is needed to refine these relationships.

Burkholderia spp. are responsible for a number of potentially devastating infectious diseases for which no vaccines currently exist. The presence of a wide variety of bacteriophages within these bacteria opens the possibility that phage therapy may be developed to augment present antibiotic treatments. We present here a detailed comparative analysis of gene content within and between groups of bacteriophages, putative prophages, and prophage-like regions in various Burkholderia species and strains. Several interesting genes and gene groups associated with pathogenicity and various metabolic functions were identified within specific groups. This study provides the first estimate of the relative contribution of prophages to the vast phenotypic diversity found among the Burkholderiae.

\section{Additional material}

Additional file 1: Additional tables. This file contains Tables S1 and S2 that describe the host range of phiE202 and all the strains that were used to search for prophages. Table S1. Bacterial strains used to examine the host range of bacteriophage phiE202. Table S2. Burkholderia strains searched for putative prophage.

\begin{abstract}
Acknowledgements
This research was sponsored by the Medical Biological Defense Research Program, U.S. Army Medical Research and Materiel Command (project 02-45X-026). This project was also funded with federal funds from the National Institute of Allergy and Infectious Diseases, National Institutes of Health, Department of Health and Human Services under contract number N01-AI30071.

We thank Kathy Kuehl for assistance with electron microscopy. The opinions, interpretations, conclusions, and recommendations expressed here are those of the author and are not necessarily endorsed by the U.S. Army in accordance with AR 70-31.
\end{abstract}

\section{Author details}

1J. Craig Venter Institute, 9704 Medical Center Drive, Rockville, MD 20850, USA. ${ }^{2}$ U.S. Army Medical Research Institute of Infectious Diseases, 1425 Porter Street, Fort Detrick, MD 21702, USA. ${ }^{3}$ Department of Microbiology, University of Georgia, Athens, GA 30602, USA. ${ }^{4}$ U.S. Department of Energy, Office of Biological and Environmental Research, SC-23.2/Germantown Building, 1000 Independence Avenue SW, Washington DC 20585-1290, USA.

\begin{abstract}
Authors' contributions
CMR and LL conducted data analyses, comparative genomics, and wrote manuscript. LB and Jl participated in bioinformatic and genomic analysis. RU and DD isolated and characterize phages and isolated phage DNA. MS isolated RNA for transcritpome analysis. WCN and DD conceived of the study, participated in its design and coordination, and helped draft manuscript. All authors have read and approved the final manuscript.
\end{abstract}

Received: 15 April 2010 Accepted: 28 July 2010 Published: 28 July 2010

\section{References}

1. Rotz LD, Khan AS, Lillibridge SR, Ostroff SM, Hughes JM: Public health assessment of potential biological terrorism agents. Emerg Infect Dis 2002, 8(2):225-230.
2. Vietri N, DeShazer D: Melioidosis. Medical Aspects of Biological Warfare Washington, DC: Dept of the Army, Office of the Surgeon General, Borden InstituteDembek Z 2007, 225-230.

3. Holden MT, Titball RW, Peacock SJ, Cerdeno-Tarraga AM, Atkins T, Crossman LC, Pitt T, Churcher C, Mungall K, Bentley SD, et al: Genomic plasticity of the causative agent of melioidosis, Burkholderia pseudomallei. Proc Natl Acad Sci USA 2004, 101(39):14240-14245.

4. Tuanyok A, Leadem BR, Auerbach RK, Beckstrom-Sternberg SM, BeckstromSternberg JS, Mayo M, Wuthiekanun V, Brettin TS, Nierman WC, Peacock SJ, et al: Genomic islands from five strains of Burkholderia pseudomallei. BMC Genomics 2008, 9:566.

5. Tumapa S, Holden MT, Vesaratchavest M, Wuthiekanun V, Limmathurotsakul D, Chierakul W, Feil EJ, Currie BJ, Day NP, Nierman WC, et al: Burkholderia pseudomallei genome plasticity associated with genomic island variation. BMC Genomics 2008, 9:190.

6. DeShazer D: Genomic diversity of Burkholderia pseudomallei clinical isolates: subtractive hybridization reveals a Burkholderia mallei-specific prophage in B. pseudomallei 1026b. J Bacteriol 2004, 186(12):3938-3950.

7. Waag DM, DeShazer D: Glanders: New Insights into an Old Disease. Biological Weapons Defense: Infectious Diseases and Counterbioterrorism Totowa, NJ: Humana Press, IncLindler LE, Lebeda FJ, Korch GW 2004

8. Losada L, Ronning CM, DeShazer D, Woods D, Kim HS, Fedorova N, Shabalina SA, Tan P, Nandi T, Pearson T, et al: Continuing evolution of Burkholderia mallei through genome reduction and large scale rearrangements. Genome Biol Evol 2010, 2010:102-116.

9. Nierman WC, DeShazer D, Kim HS, Tettelin H, Nelson KE, Feldblyum T, Ulrich RL, Ronning CM, Brinkac LM, Daugherty SC, et al: Structural flexibility in the Burkholderia mallei genome. Proc Natl Acad Sci USA 2004, 101(39):14246-14251.

10. Brett PJ, DeShazer D, Woods DE: Burkholderia thailandensis sp. nov., a Burkholderia pseudomallei-like species. Int J Syst Bacteriol 1998, 48(Pt 1):317-320.

11. Smith MD, Angus BJ, Wuthiekanun V, White NJ: Arabinose assimilation defines a nonvirulent biotype of Burkholderia pseudomallei. Infect Immun 1997, 65(10):4319-4321.

12. Moore RA, Reckseidler-Zenteno S, Kim H, Nierman W, Yu Y, Tuanyok A, Warawa J, Deshazer D, Woods DE: Contribution of gene loss to the pathogenic evolution of Burkholderia pseudomallei and Burkholderia mallei. Infect Immun 2004, 72(7):4172-4187.

13. Mahenthiralingam E, Baldwin A, Dowson CG: Burkholderia cepacia complex bacteria: opportunistic pathogens with important natural biology. J App/ Microbiol 2008, 104(6):1539-1551.

14. Figueroa-Bossi N, Uzzau S, Maloriol D, Bossi L: Variable assortment of prophages provides a transferable repertoire of pathogenic determinants in Salmonella. Mol Microbiol 2001, 39(2):260-271.

15. Ventura M, Canchaya C, Pridmore D, Berger B, Brussow H: Integration and distribution of Lactobacillus johnsonii prophages. J Bacteriol 2003, 185(15):4603-4608

16. Ventura M, Canchaya C, Bernini V, Altermann E, Barrangou R, McGrath S, Claesson MJ, Li Y, Leahy S, Walker CD, et al: Comparative genomics and transcriptional analysis of prophages identified in the genomes of Lactobacillus gasseri, Lactobacillus salivarius, and Lactobacillus casei. Appl Environ Microbiol 2006, 72(5):3130-3146.

17. Nakagawa I, Kurokawa K, Yamashita A, Nakata M, Tomiyasu Y, Okahashi N, Kawabata S, Yamazaki K, Shiba T, Yasunaga T, et al: Genome sequence of an M3 strain of Streptococcus pyogenes reveals a large-scale genomic rearrangement in invasive strains and new insights into phage evolution. Genome Res 2003, 13(6A):1042-1055.

18. Van Sluys MA, de Oliveira MC, Monteiro-Vitorello CB, Miyaki CY, Furlan LR, Camargo LE, da Silva AC, Moon DH, Takita MA, Lemos EG, et al: Comparative analyses of the complete genome sequences of Pierce's disease and citrus variegated chlorosis strains of Xylella fastidiosa. J Bacteriol 2003, 185(3):1018-1026.

19. Boyd EF, Brussow H: Common themes among bacteriophage-encoded virulence factors and diversity among the bacteriophages involved. Trends Microbiol 2002, 10(11):521-529.

20. Hendrix RW, Lawrence JG, Hatfull GF, Casjens S: The origins and ongoing evolution of viruses. Trends Microbiol 2000, 8(11):504-508.

21. Woods DE, Jeddeloh JA, Fritz DL, DeShazer D: Burkholderia thailandensis E125 harbors a temperate bacteriophage specific for Burkholderia mallei. J Bacteriol 2002, 184(14):4003-4017. 
22. Lech K, Brent R: Plating lambda phage to generate plaques. Current Protocols in Molecular Biology New York: John Wiley \& SonsAusubel FM, Brent R, Kingston RE, Moore DD, Seidman JG, Smith JA, Struhl K 1987, 1.11.11-11.11.14

23. Lin X, Kaul S, Rounsley S, Shea TP, Benito MI, Town CD, Fujii CY, Mason T, Bowman CL, Barnstead M, et al: Sequence and analysis of chromosome 2 of the plant Arabidopsis thaliana. Nature 1999, 402(6763):761-768.

24. Yu Y, Kim HS, Chua HH, Lin CH, Sim SH, Lin D, Derr A, Engels R, DeShazer D, Birren B, et al: Genomic patterns of pathogen evolution revealed by comparison of Burkholderia pseudomallei, the causative agent of melioidosis, to avirulent Burkholderia thailandensis. BMC Microbiol 2006, 6:46.

25. Chain PS, Denef VJ, Konstantinidis KT, Vergez LM, Agullo L, Reyes VL, Hauser L, Cordova M, Gomez L, Gonzalez M, et al: Burkholderia xenovorans LB400 harbors a multi-replicon, 9.73-Mbp genome shaped for versatility. Proc Natl Acad Sci USA 2006, 103(42):15280-15287.

26. Canchaya C, Proux C, Fournous G, Bruttin A, Brussow H: Prophage genomics. Microbiol Mol Biol Rev 2003, 67(2):238-276, table of contents.

27. Casjens S: Prophages and bacterial genomics: what have we learned so far? Mol Microbiol 2003, 49(2):277-300

28. Altschul SF, Lipman DJ: Protein database searches for multiple alignments. Proc Natl Acad Sci USA 1990, 87(14):5509-5513.

29. Summer EJ, Gonzalez CF, Carlisle T, Mebane LM, Cass AM, Savva CG, LiPuma J, Young R: Burkholderia cenocepacia phage BcepMu and a family of Mu-like phages encoding potential pathogenesis factors. J Mol Biol 2004, 340(1):49-65.

30. Summer EJ, Gonzalez CF, Bomer M, Carlile T, Embry A, Kucherka AM, Lee J, Mebane L, Morrison WC, Mark L, et al: Divergence and mosaicism among virulent soil phages of the Burkholderia cepacia complex. J Bacteriol 2006, 188(1):255-268.

31. Sonnhammer EL, Durbin R: A dot-matrix program with dynamic threshold control suited for genomic DNA and protein sequence analysis. Gene 1995, 167(1-2):GC1-10.

32. Rohwer F, Edwards R: The Phage Proteomic Tree: a genome-based taxonomy for phage. J Bacteriol 2002, 184(16):4529-4535.

33. Felsenstein J: PHYLIP (Phylogeny Inference Package), version 3.6. Department of Genome Sciences, University of Washington, Seattle 2005, Distributed by the author.

34. Darling ACE, Mau B, Blattner FR, Perna NT: Mauve: multiple alignment of conserved genomic sequence with rearrangements. Genome Res 2004 14(7):1394-1403.

35. Studholme DJ, Dixon R: Domain architectures of sigma 54-dependent transcriptional activators. J Bacterio/ 2003, 185(6):1757.

36. Reese MG: Application of a time-delay neural network to promoter annotation in the Drosophila melanogaster genome. Comput Chem 2001, 26(1):51-56.

37. Kingsford C, Ayanbule K, Salzberg S: Rapid, accurate, computational discovery of Rho-independent transcription terminators illuminates their relationship to DNA uptake. Genome Biol 2007, 8(2):R22.

38. Ackermann HW: Bacteriophage observations and evolution. Res Microbiol 2003, 154(4):245-251

39. DeShazer D, Waag DM, Fritz DL, Woods DE: Identification of a Burkholderia mallei polysaccharide gene cluster by subtractive hybridization and demonstration that the encoded capsule is an essential virulence determinant. Microb Pathog 2001, 30(5):253-269.

40. Brussow H, Hendrix RW: Phage genomics: small is beautiful. Cell 2002, 108(1):13-16.

41. Hendrix RW, Hatfull GF, Smith MC: Bacteriophages with tails: chasing their origins and evolution. Res Microbiol 2003, 154(4):253-257.

42. Summer EJ, Gill JJ, Upton C, Gonzalez CF, Young R: Role of phages in the pathogenesis of Burkholderia, or 'Where are the toxin genes in Burkholderia phages?'. Curr Opin Microbiol 2007, 10(4):410-417.

43. Hayes F: Toxins-antitoxins: plasmid maintenance, programmed cell death, and cell cycle arrest. Science 2003, 301(5639):1496-1499.

44. Labrie SJ, Josephsen J, Neve H, Vogensen FK, Moineau S: Morphology, genome sequence, and structural proteome of type phage P335 from Lactococcus lactis. Appl Environ Microbiol 2008, 74(15):4636-4644.

45. Ikebe T, Wada A, Inagaki $Y$, Sugama K, Suzuki R, Tanaka D, Tamaru A, Fujinaga $Y$, Abe $Y$, Shimizu $Y$, et al: Dissemination of the phage-associated novel superantigen gene speL in recent invasive and noninvasive
Streptococcus pyogenes M3/T3 isolates in Japan. Infect Immun 2002, 70(6):3227-3233.

46. Brussow H, Desiere F: Comparative phage genomics and the evolution of Siphoviridae: insights from dairy phages. Mol Microbiol 2001, 39(2):213-222.

47. Juhala RJ, Ford ME, Duda RL, Youlton A, Hatfull GF, Hendrix RW: Genomic sequences of bacteriophages HK97 and HK022: pervasive genetic mosaicism in the lambdoid bacteriophages. J Mol Biol 2000, 299(1):27-51.

48. Buchen-Osmond C: ICTVdB - The Universal Virus Database, version 3. Columbia University, New York 2006.

49. Sitkiewicz I, Stockbauer KE, Musser JM: Secreted bacterial phospholipase A2 enzymes: better living through phospholipolysis. Trends Microbiol 2007, 15(2):63-69.

50. Pukatzki S, Kessin RH, Mekalanos JJ: The human pathogen Pseudomonas aeruginosa utilizes conserved virulence pathways to infect the social amoeba Dictyostelium discoideum. Proc Natl Acad Sci USA 2002, 99(5):3159-3164

51. Sacks DL, Modi G, Rowton E, Späth G, Epstein L, Turco SJ, Beverley SM: The role of phosphoglycans in Leishmania-sand fly interactions. Proc Natl Acad Sci USA 2000, 97(1):406-411.

52. Woods DE: The use of animal infection models to study the pathogenesis of melioidosis and glanders. Trends Microbiol 2002, 10(11):483-484, discussion 484-485.

doi:10.1186/1471-2180-10-202

Cite this article as: Ronning et al:: Genetic and phenotypic diversity in Burkholderia: contributions by prophage and phage-like elements. BMC Microbiology 2010 10:202.

\section{Submit your next manuscript to BioMed Central and take full advantage of:}

- Convenient online submission

- Thorough peer review

- No space constraints or color figure charges

- Immediate publication on acceptance

- Inclusion in PubMed, CAS, Scopus and Google Scholar

- Research which is freely available for redistribution
C Biomed Central 